\title{
Los adolescentes y el conflicto interparental destructivo: impacto en la percepción del sistema familiar y diferencias según el tipo de familia, la edad y el sexo de los adolescentes"
}

\author{
Adolescents and Destructive Interparental Conflict: \\ Impact on the Perception of the Family System and Differences \\ by Adolescents' Type of Family, Age and Sex
}

Recibido: octubre 10 de 2011 | Revisado: marzo 23 de 2012 | Aceptado: mayo 28 de 2012

\author{
SILVIA LÓPEZ LARROSA** \\ VANESA SÁNCHEZ SOUTO *** \\ PAUla MENDIRI Ruíz DE AldA ${ }^{* * * *}$ \\ Universidade da Coruña, España
}

SICI: 2011-2277(201212)11:4<1255:LAYCID>2.0.CO;2-5

Para citar este artículo: López Larrosa, S., Sánchez Souto, V. \& Mendiri, P. (2012). Los adolescentes y el conflicto interparental destructivo: impacto en la percepción del sistema familiar y diferencias según el tipo de familia, la edad y el sexo de los adolescentes. Universitas Psychologica, 11(4), 1255-1262.

Esta investigación hace parte del trabajo previo a la elaboración de la tesis doctoral de Vanesa Sánchez Souto.

** Profesora titular del Departamento de Psicología Evolutiva y de la Educación. E-mail:silvia@udc.es

**** Alumna de doctorado en el Departamento de Psicología Evolutiva y de la Educación. E-mail: tecvss01@udc.es

******* Profesora titular del Departamento de Filosofía y Métodos de investigación en Educación. E-mail: paulamen@udc.es
RESUMEN

En este trabajo se estudiaron las percepciones del conflicto interparental y sus efectos a largo plazo en las emociones, las conductas y las cogniciones en una muestra de 510 adolescentes españoles. Los instrumentos empleados fueron la Children's Perception of Interparental Conflict Scale [CPIC] (Grych, Seid \& Fincham, 1992) y la Security in the Family System Scale [SIFS] (Forman \& Davies, 2005). CPIC evalúa la naturaleza del conflicto y los factores contextuales del mismo. SIFS mide la seguridad en el sistema familiar, la preocupación y la implicación de los hijos. Se encontró que cuanto más conflicto destructivo hay entre los padres (más intenso, frecuente y sin resolver) más culpados, amenazados, triangulados, preocupados e inseguros se sienten los hijos, más ineficaces piensan que son sus intentos de resolver los problemas de los padres y menos se implican en las discusiones. Se encontraron diferencias entre familias monoparentales y nucleares que hacen patente el impacto del conflicto posdivorcio de los padres. En estos casos es más estable y los padres son más ineficaces en su resolución, por lo que los hijos se sienten más inseguros. Los datos indican que con la edad aumenta la inseguridad de los hijos y que las jóvenes se implican significativamente menos en los conflictos entre los padres.

Palabras clave autores

Conflicto familiar, adolescentes, impacto.

Palabra clave descriptores

Conflicto interparental destructivo, adolescentes, seguridad emocional.

\begin{abstract}
A B S T R A C T
We studied the perceptions of interparental conflict and its long term effects on emotions, behaviours and cognitions in a sample of 510 Spanish adolescents. The instruments used were the Children's Perception of Interparental Conflict Scale [CPIC] (Grych, Seid \& Fincham, 1992) and the Security in the Family System Scale [SIFS] (Forman \& Davies, 2005). CPIC evaluates the nature of conflict and its contextual variables. SIFS measures the security in the family system, the preoccupation and the disengagement of children. We have found that the more destructive interparental conflict happens (more intense, frequent and unsolved), more guilty, threatened, triangulized, worried and insecure children feel, more useless their attempts
\end{abstract}


to solve parental problems are and more disengage from interparental disagreements. There are differences between single mother families and nuclear families. These depict that post divorce conflict has a great impact on children. In this case, it is more stable and parents are less efficient in solving it, so children feel more insecure. Our data show that, with age, there is an increase in children's insecurity and that girls disengage more than boys when facing interparental conflict. Key words authors

Family Conflict, Adolescents, Impact.

Key words plus

Destructive Interparental Conflict, Adolescents, Emotional Security.

Según Cummings y Davies (2010), el conflicto matrimonial puede definirse como "cualquier interacción entre los padres (...) que implica una diferencia de opinión, tanto si es fundamentalmente negativa como incluso fundamentalmente positiva" (p. 8). De este modo, se incluyen dentro de esta definición un amplio abanico de comportamientos: agresión física, agresión verbal y no verbal, defensividad, retirada y también apoyo, afecto y solución de problemas. No obstante, el tipo de conflicto que más bibliografía ha generado es el que se sitúa en el lado más negativo, el de la agresión, la hostilidad y la descalificación, que se prolonga en el tiempo y que no se resuelve. Con respecto a este conflicto entre los padres, que se ha denominado conflicto destructivo, las investigaciones han puesto en evidencia los efectos a corto y largo plazo sobre los hijos en las respuestas fisiológicas, conductuales, emocionales y cognitivas (Cantón, Cortés \& Justicia, 2007; Cummings \& Davies, 1994, 2010; López Larrosa, 2009).

El presente trabajo se centrará esencialmente en las respuestas emocionales, conductuales y cognitivas a largo plazo, utilizando para ello dos de los modelos más potentes de explicación del impacto del conflicto interparental sobre los hijos: el modelo cognitivo-contextual (Grych \& CardozaFernandes, 2001; Grych \& Fincham, 1990) y la teoría de la seguridad emocional (Cummings \& Davies, 2010; Davies, Harold, Goeke-Morey \& Cummings, 2002).

Según el primer modelo, los hijos evalúan si el conflicto es relevante (esto es, si ellos "tienen la culpa") o amenazante (por estar en medio o sentirse ineficaces para actuar), y después por qué ocurre y cómo responder a él. Dichas evaluaciones están afectadas por las propiedades específicas del conflicto como su intensidad, frecuencia, estabilidad y resolución, y por factores contextuales, especialmente su edad, su sexo y las experiencias previas con el conflicto (generalmente muy vinculadas con la edad, porque a mayor edad, más probabilidad de haber presenciado conflictos entre los padres).

Según la teoría de la seguridad emocional, una meta central para los niños es mantener y promover el sentirse protegidos en su familia (seguridad emocional). Cuando los hijos están expuestos al conflicto destructivo de sus padres, se sensibilizan y buscan preservar la seguridad emocional en contextos de conflicto subsiguientes. Dado que la preservación de la seguridad emocional es una meta tan destacada, la amenaza que supone el conflicto destructivo incrementa su preocupación $\mathrm{y}$, por tanto, su reactividad al conflicto entre sus padres. Cuando en la familia hay conflictos, los niños usan estrategias inseguras que consisten en estar preocupados para estar listos e identificar pronto cualquier amenaza a su bienestar, o desvincularse de su familia para disminuir la importancia que esta tiene en sus vidas. Por tanto, seguridad, preocupación y respuesta de los niños son las tres dimensiones más relevantes desde esta teoría.

Tomando en consideración ambos modelos, se partió de identificar como especialmente relevantes la intensidad y frecuencia del conflicto. Aunque estas pueden ser percibidas de modo distinto por los adolescentes -moduladas por el resto de características que evalúan el modelo cognitivocontextual y la teoría de la seguridad emocional-, parecen las dimensiones más estructurales del conflicto y más objetivamente evaluables por los hijos. Pero, es preciso ver su relación con otras propiedades específicas, especialmente la estabilidad y resolución. Esta última tiene una importancia crucial en la diferenciación entre conflicto constructivo y destructivo. Si los chicos ven que sus padres llegan a acuerdos, y las diferencias se resuelven, esta resolución satisfactoria permite 
caracterizar al conflicto como constructivo y constituye una importante fuente de aprendizaje positivo para sus hijos. Si, por el contrario, no ven que lleguen a acuerdos, el conflicto es destructivo. Por otra parte, si los conflictos se manifiestan preferentemente de una determinada manera y son muy estables a lo largo del tiempo, su efecto positivo o negativo se ve potenciado.

El modelo cognitivo-contextual también propone que los factores contextuales son importantes. De estos se seleccionaron la edad y el sexo, dado que la edad está muy vinculada a las experiencias previas de conflicto, que es la otra variable contextual del modelo. Con respecto a la edad, al incrementar y mejorar sus capacidades de pensamiento abstracto, los adolescentes son más capaces de inferir y evaluar las implicaciones personales de la calidad de la vida familiar y de los conflictos interparentales. En la adolescencia, como los hijos han estado expuestos a los problemas adultos durante más tiempo, son más sensibles al conflicto interparental (Cummings, Schermerhorn, Davies, Goeke-Morey \& Cummings, 2006) y a formas sutiles de interacciones familiares. Discriminan mejor, por ejemplo, entre conflicto constructivo y destructivo (Bickham \& Fiese, 1997; Davies \& Cummings, 1998), por lo que resultan más afectados que los niños más pequeños (Justicia \& Cantón, 2011). Por esto, se estudiaron adolescentes más jóvenes y mayores; aunque en un estudio realizado en España por Rodrigo, Máiquez, Padrón y García-Ruiz (2009) no se hallaron diferencias en las atribuciones realizadas al conflicto al comparar adolescentes de diversas edades.

Por lo que respecta al papel del sexo, las investigaciones (Amato, 2000; Cummings \& Davies, 2010) no se ponen de acuerdo. Mientras en unas sí se han encontrado diferencias entre chicos y chicas en el impacto del conflicto y en su respuesta a él, en otras no. Con respecto al impacto del conflicto, algunos estudios indican que los varones ven los conflictos negativos entre sus padres más como una amenaza percibida, mientras que en las mujeres se produce más autoinculpación (Cummings, GoekeMorey \& Papp, 2004). En cuanto a las respuestas ante el conflicto, en las adolescentes se ha encontrado o mayor involucración o evitación (Davies et al., 2002; El-Sheikh \& Reiter, 1996; Goeke-Morey, Cummings, Harold \& Shelton, 2003), y más empeño en restaurar la armonía interparental (Davies \& Lindsay, 2004). En otros trabajos, el impacto del conflicto es el mismo, pero la respuesta difiere. Así, según Jouriles y Norwood (1995) varones y mujeres experimentan el mismo nivel de estrés ante los conflictos de sus padres, pero las chicas reaccionan de forma pasiva o con buena conducta para reducir el conflicto y provocar cambios positivos en la familia (Luthar, Doernberger \& Zigler, 1993).

Finalmente, para otros las diferencias tienen más que ver con la diferente socialización de los niños (Cummings \& Davies, 1994). Esto se manifiesta en resultados a veces contradictorios en los que, por una parte, los padres son más tolerantes con la agresividad y el mal comportamiento de los hijos (Lytton, 1991) y, por otra, están expuestos a mayor negatividad e insensibilidad de sus progenitores (Kurdek, 1986; Rutter, 1990).

Según la teoría de la seguridad emocional, dado que la meta de la seguridad es esencial para los hijos, esta también deberá evaluarse, junto con la preocupación y la respuesta al conflicto entre los padres, como se plantea en la SIFS. Este instrumento introduce, con respecto a la CPIC, un matiz importante, y es que está concebido para evaluar a todo el sistema familiar. Esto implica que los hijos pueden percibir otras fuentes de seguridad o inseguridad, además de los padres, tenga las características estructurales que tenga el sistema familiar. Pero, como señalan Cummings y Davies (2010), se han hecho muy pocas investigaciones en diferentes formas (estructuras) familiares y, en concreto, plantean la importancia de estudiar los procesos de conflicto y sus efectos en los niños en las familias monoparentales, en las que los padres no viven juntos pero siguen relacionándose. Por ello, en este trabajo se explora también la existencia de diferencias entre jóvenes de familias monoparentales y nucleares, como dos formas familiares estructuralmente diferentes.

Se pretende, por tanto, evaluar el papel de la naturaleza del conflicto interparental en las cogni- 
ciones, las emociones y la respuesta de los hijos, modulados por los factores contextuales identificados por el modelo cognitivo-contextual (edad y sexo) y las características estructurales de la familia. De modo más específico, nuestros objetivos son:

1. Identificar las relaciones entre la intensidad-frecuencia del conflicto y la naturaleza del conflicto, las cogniciones, las emociones y la conducta de los adolescentes evaluadas por el modelo cognitivocontextual y la teoría de la seguridad emocional.

2. Identificar las relaciones entre la edad y el sexo y las características estructurales del sistema familiar (familia nuclear-familia monoparental) en las cogniciones, las emociones y la conducta de los adolescentes.

\section{Método}

\section{Participantes}

En esta investigación participaron 510 alumnos de educación pública residentes en la ciudad de Lugo (España). Sus edades estaban comprendidas entre los 11 y los 18 años, y tenían una edad media de 14.86 años $(D E=1.77)$. Un poco más de la mitad (54\%) eran mujeres y $46 \%$ eran varones.

La distribución por cursos fue bastante equilibrada entre 1.. de ESO (Enseñanza Secundaria Obligatoria) y 2. de bachillerato: un $13.6 \%$ estudiaba 1.. de ESO, el $13.8 \%$ estaba en 2.o, el $18.7 \%$

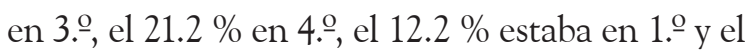
$20.6 \%$ en 2. de bachillerato.

Con respecto a la estructura familiar, la mayoría (84.5\%) vivía con ambos progenitores, un $12.8 \%$, en familias monoparentales y un $2.8 \%$, en otras configuraciones familiares. La media de miembros de las familias fue de $3.97(D E=1.074)$. Tanto ellos $(94.3 \%)$ como sus padres $(92.6 \%)$ y madres $(90.6 \%)$ eran españoles.

\section{Instrumentos}

Según Cummings y Davies (2010), los hijos son mejores fuentes de información sobre la destructividad o constructividad del conflicto que los padres u observadores externos. Los instrumentos utilizados recurren a ellos y están vinculados a los dos modelos teóricos explicativos del impacto del conflicto en la familia, expuestos anteriormente.

\section{The Children's Perception of Interparental} Conflict Scale [CPIC] (Grych et al., 1992)

En el presente trabajo se utilizó la versión española traducida y adaptada como Escala de Conflicto Interparental desde la Perspectiva de los Hijos por Martínez-Pampliega (2008). La CPIC es un instrumento que evalúa la percepción del conflicto entre los padres. Incluye nueve subescalas: frecuencia, contenido, intensidad, resolución, amenaza percibida, eficacia de afrontamiento, autoculpa, triangulación y estabilidad causal. En su versión original consta de 49 ítems y en la versión traducida y adaptada, de 36 ítems. Tiene tres opciones de respuesta: $1=$ verdadero, $2=$ casi verdadero y $3=$ falso.

Los análisis realizados con los participantes en el estudio permitieron extraer siete factores que explican el $63.96 \%$ de la varianza. Un factor agrupa Autoculpa y Contenido ("mis padres suelen discutir sobre cosas que he hecho yo") y otro agrupa Intensidad y Frecuencia ("mis padres gritan cuando discuten" o "a menudo veo a mis padres discutir"). El resto de los factores se ajustan a lo encontrado por Martínez-Pampliega y a la versión original de Grych et al. (1992): Resolución ("mis padres suelen encontrar una solución después de discutir"), Amenaza Percibida ("cuando mis padres discuten temo que algo malo me ocurra"), Eficacia de Afrontamiento ("cuando mis padres discuten no puedo hacer nada para sentirme mejor"), Triangulación ("creo que debo apoyar a uno de los dos cuando mis padres riñen") y Estabilidad Causal ("mis padres discuten porque realmente no se quieren"). El valor del alfa de Cronbach de la escala total con esta muestra es de 0.92 y para cada subescala es superior a 0.8 , excepto en Eficacia de Afrontamiento (0.8) y en Triangulación (0.51) (Sánchez Souto, López Larrosa \& Mendiri, 2010). 
Versión española de la Security in the Family System Scale [SIFS] (Forman \& Davies, 2005)

La SIFS evalúa la percepción de seguridad que tienen los niños y adolescentes en la familia como un todo. Puede administrarse a partir de los 10 años. Consta de 24 ítems con 5 alternativas de respuesta, desde muy en desacuerdo (1) a muy de acuerdo (5). El cuestionario fue traducido por las autoras y por nativos ingleses respetando los criterios de traducción inversa.

En la versión inglesa se extrajeron tres factores: Preocupación, Seguridad y Falta de Compromiso, que explican el $55.4 \%$ de la varianza. En esta versión española también se extrajeron los mismos factores, que explican el $53.25 \%$ de la varianza. El alfa de Cronbach para toda la escala en la muestra del estudio fue de 0.91. La Preocupación ("tengo la sensación de que mi familia tendrá muchos cambios que yo no espero"), la Seguridad ("creo que los miembros de mi familia estarán cerca para ayudarme en el futuro”) y la Falta de Compromiso o implicación ("cuando pasa algo malo en mi familia, me gustaría vivir en una familia diferente") tienen unos valores de consistencia interna superiores a 0.84 , menos en el caso de la Falta de Implicación, cuyo valor de alfa de Cronbach es de 0.78 (Sánchez Souto et al., 2010).

\section{Procedimiento}

Se solicitó el permiso para aplicar los cuestionarios. Desde la dirección se consultó a los docentes y estos informaron a los alumnos para pedir el consentimiento de los padres. Una vez obtenidos los consentimientos, se procedió a aplicar los cuestionarios de modo grupal, en el tiempo en que los alumnos estaban con su tutor o algún docente que expresamente hubiese indicado que permitía la aplicación de los cuestionarios durante la hora asignada a su asignatura. En todos los casos estuvo presente una de las investigadoras de este trabajo. Los alumnos debían contestar anónimamente primero unas preguntas básicas sociodemográficas (edad, sexo o número de miembros en la familia) y después cada uno de los cuestionarios, primero la CPIC y después la SIFS.
Para el análisis de datos se empleó el programa estadístico SPSS versión 15.0. Se seleccionó inicialmente la variable intensidad y frecuencia del conflicto como variable independiente. Para analizar el papel de los factores contextuales edad y sexo, y de la estructura familiar, se introdujeron también como variables independientes. Las variables dependientes fueron estabilidad y resolución (variables: naturaleza del conflicto), contenido y culpa (variables cognitivas de evaluación del conflicto), amenaza, eficacia y triangulación (variables cognitivas de amenaza y eficacia), preocupación y seguridad (variables emocionales) y falta de implicación y estrategias de afrontamiento (variables conductuales). Con estas variables se realizaron regresiones para datos categóricos (escalamiento óptimo).

Una vez realizadas las regresiones, se hicieron ANOVA de un factor con las variables estadísticamente significativas en la regresión.

\section{Resultados}

Los análisis de regresión fueron estadísticamente significativos con todos los factores analizados. Por lo que respecta a las variables de naturaleza del conflicto, en el factor estabilidad $(F(8.465)=84.63$, $p<0.001)$ las variables independientes explican el $59.3 \%$ de la varianza, siendo las variables más relevantes la intensidad y frecuencia del conflicto $(p<0.001)$, que explica el $39 \%$ de la varianza; y el tipo de familia $(p<0.001)$, que explica el $9 \%$ de la varianza. En el factor resolución $(F(4.467)<55.39$, $p<0.001)$, las variables independientes explican el $45 \%$ de la varianza, siendo las más relevantes la intensidad y frecuencia del conflicto $(p<0.001)$, que explica el $28 \%$ de la varianza, y el tipo de familia $(p<0.001)$, que explica el $6 \%$.

Por lo que respecta a las variables cognitivas, para contenido y culpa $(F(7.469)=136.49 ; p<0.001)$, las variables independientes explican el $67 \%$ de la varianza pero sin duda es la intensidad y frecuencia del conflicto la variable más relevante $(p<0.001)$, puesto que ella sola explica el $65 \%$ de la varianza. En el factor amenaza $(F(6.470)=64.99, p<0.001)$, las variables independientes explican el $45 \%$ de 
la varianza y nuevamente la variable más relevante es la intensidad y frecuencia del conflicto $(p<0.001)$, que explica ella sola el $43.56 \%$ de la varianza. En el factor eficacia $(F(9.467)=20.80$, $p<0.001)$, las variables independientes explican el $28 \%$ de la varianza; siendo las más relevantes, nuevamente, la intensidad y frecuencia del conflicto $(p<0.001)$, que explica el $26.83 \%$ de la varianza y la edad $(p<0.001)$, que explica el $1.3 \%$. En el factor triangulación $(F(7.467)=15.91, p<0.001)$, las variables independientes explican el $19.3 \%$ de la varianza; siendo las más relevantes la intensidad y frecuencia del conflicto $(p<0.001)$, que explica el $17.13 \%$ de la varianza, y el sexo $(p<0.001)$, que explica el $1.2 \%$ de la varianza.

En cuanto a los factores emocionales, en preocupación $(F(7,467)=24.16, p<0.001)$, las variables independientes explican el $26.6 \%$ de la varianza, siendo las más relevantes la intensidad y frecuencia del conflicto $(p<0.001)$, que explica el $20.61 \%$ de la varianza, y el tipo de familia $(p<0.001)$, que explica el $2.1 \%$. En seguridad $(F(7,467)=17.05$, $p<0.001$ ), las variables independientes explican el $20.4 \%$ de la varianza, siendo las más relevantes la intensidad y frecuencia del conflicto $(p<0.001)$, que explica el $13.98 \%$ de la varianza y la edad $(p<0.0012)$, que explica el $1.69 \%$.

Finalmente, por lo que respecta a las variables conductuales, para falta de implicación $(F(6.468)=23.57, p<0.001)$, las variables independientes explican el $23.57 \%$ de la varianza, siendo las más relevantes la intensidad y frecuencia del conflicto $(p<0.001)$, que explica el $19 \%$ de la varianza, y el sexo $(p<0.0026)$, que explica el $0.8 \%$.

Dado el papel de cada una de las variables independientes, con respecto a las variables de naturaleza del conflicto y a las cogniciones, emociones y conductas, se analizó cada una de dichas variables independientes por separado. Para la intensidad y frecuencia del conflicto, se seleccionaron los sujetos cuyos valores de intensidad y frecuencia eran más extremos (percentiles 25 y 75), indicando o mucha intensidad y frecuencia del conflicto o muy baja. De este modo, la muestra inicial se redujo a 282 sujetos. Con ellos se realizaron análisis de varianza de un factor con cada una de las variables depen- dientes. Los datos, estadísticamente significativos $(p<0.01)$, pusieron de manifiesto que a mayor intensidad y frecuencia del conflicto, más atribuciones a la estabilidad y más ineficaces las soluciones de los padres (naturaleza del conflicto), más elevados los valores de contenido y culpa (cogniciones: evaluación del conflicto), más amenazados se sentían los adolescentes, más sensación de ineficacia por su parte, más triangulación (cogniciones: amenaza y eficacia para actuar), y más preocupación e inseguridad (emociones) y falta de implicación (conducta) de los hijos.

Se hicieron ANOVA con la variable tipo de familia para aquellos factores que fueron significativos en el escalamiento. Para distinguir entre chicos que vivían con ambos progenitores y los que vivían solo con la madre, la muestra debió reducirse a 493 sujetos, de los cuales 63 vivían con sus madres solas y 430 con padre y madre. Los análisis fueron estadísticamente significativos $(p<0.01)$, de modo que los chicos que viven solo con sus madres, comparados con los que viven con ambos progenitores, hacen unas atribuciones mayores a la estabilidad, más ineficaces consideran que son las soluciones de sus padres (naturaleza del conflicto) y más preocupados están (emociones).

La variable edad, que resultó significativa con la variable eficacia y con seguridad, se dicotomizó para distinguir entre el grupo de chicos iguales o menores de 14 años $(N=209)$ y mayores de 15 años $(N=296)$. En este caso, solo resultó significativa la seguridad $(p<0.001)$, de modo que los que sienten más inseguridad en su familia son los chicos mayores.

Finalmente, con la variable sexo, que resultó significativa en el escalamiento, con respecto a las variables triangulación y falta de implicación, se realizaron ANOVA y solo resultó significativa la falta de implicación $(p<0.001)$. La media de falta de implicación de las chicas es mayor que la de los chicos.

\section{Discusión}

Los resultados ponen de manifiesto la gran relevancia que para los hijos tiene la intensidad 
y frecuencia del conflicto, superior a las otras dimensiones analizadas junto a ella como variables independientes. El impacto de la intensidad y frecuencia del conflicto se produce en las dimensiones de naturaleza del conflicto $y$, especialmente, en las emociones, las cogniciones y la conducta. A mayor intensidad y frecuencia del conflicto, más habitualmente los padres no llegan a acuerdos. Por tanto, cuando los chicos evalúan el conflicto entre sus padres, se están refiriendo especialmente a conflictos destructivos, en los que no se logran soluciones (Cummings \& Davies, 2010). Y no solo los conflictos son más frecuentes, intensos y no resueltos, sino que, además, su patrón de irresolución es estable. Ante esta situación, se producen más frecuentemente determinadas respuestas emocionales, cognitivas y conductuales de los adolescentes: se sienten amenazados pero también más culpables. No es extraño, por tanto, que aumente el sentirse en el medio (triangulados), inseguros y preocupados. Según la teoría de la seguridad emocional (Cummings \& Davies, 2010), los hijos tratan de preservar su sentido de seguridad. Por tanto, a mayor conflicto destructivo, menor seguridad y mayor preocupación, para estar listos para actuar cuando haga falta. En el presente estudio, la conducta más frecuente, precisamente, es la de falta de implicación, aquella en la que el adolescente, para preservar su sentido de seguridad según la teoría, hace ver que no le importa y no se involucra, quizá porque sus intentos de solución los perciben como más ineficaces, como también ponen de manifiesto nuestros datos.

Con respecto al papel de la estructura familiar, que, como señalan Cummings y Davies (2010) ha sido poco estudiada, los resultados parecen apuntar a que esta es más relevante que la edad y el sexo de los adolescentes. Se hallaron diferencias significativas entre las familias monoparentales y las nucleares, de modo que los chicos de familias monoparentales perciben que el conflicto entre sus padres es más estable y los padres más ineficaces. Se observó que ambas son dimensiones también muy relacionadas con la intensidad y frecuencia del conflicto. Esto indica que, pese a que los padres ya no viven juntos, los conflictos entre los progenitores continúan.
Estos conflictos posdivorcio tienen efectos más negativos en los hijos (López Larrosa, 2009). No es extraño entonces que, como indican los resultados encontrados, los hijos de familias monoparentales estén más preocupados. Nuevamente su seguridad emocional se ve afectada.

Por lo que respecta a la edad, los datos concuerdan claramente con lo apuntado por Cummings et al. (2006), poniendo en evidencia la mayor sensibilización a los problemas adultos, que en nuestro caso se traduce en una mayor inseguridad entre los chicos de más edad. Según estos autores, esto se relaciona con una historia más larga de exposición al conflicto interparental.

Finalmente, los datos relacionados con las diferencias entre chicos y chicas coinciden con lo señalado por otros autores, en la medida en que entre ellos no se dan diferencias en el impacto, en este caso, en las emociones y cogniciones evaluadas, sino en la respuesta ante el mismo (Jouriles \& Norwood, 1995; Luthar et al., 1993) que es más pasiva, de falta de implicación. Por tanto, a diferencia de lo señalado por otros investigadores, en las chicas no se da un mayor involucramiento en el conflicto, sino una mayor desvinculación como estrategia de preservación de la seguridad emocional.

\section{Referencias}

Amato, P. R. (2000). The consequences of divorce for adults and children. Journal of Marriage \& The Family, 62(4), 1269-1287.

Bickham, N. L. \& Fiese, B. H. (1997). Extension of the Children's Perceptions of Interparental Conflict Scale for use with late adolescents. Journal of Family Psychology, 11(2), 246-250.

Cantón, J., Cortés, M. R. \& Justicia, M. D. (2007). Conflictos entre los padres, divorcio y desarrollo de los hijos. Madrid: Pirámide.

Cummings, E. M. \& Davies, P. (1994). Children and marital conflict. Nueva York: Guilford Press.

Cummings, E. M. \& Davies, P. T. (2010). Marital conflict and children: An emotional security perspective. New York: The Guildford Press.

Cummings, E. M., Goeke-Morey, M. C. \& Papp, L. M. (2004). Everyday marital conflict and child 
aggression. Journal of Abnormal Child Psychology, 32(2), 191-202.

Cummings, E. M., Schermerhorn, A. C., Davies, P. T., Goeke-Morey, M. C. \& Cummings, J. S. (2006). Interparental discord and child adjustment: Prospective investigations of emotional security as an explanatory mechanism. Child Development, 77(1), 132-152.

Davies, P. T. \& Cummings, E. M. (1998). Exploring children's emotional security as a mediator of the link between marital relations and child adjustment. Child Development, 69(1), 124-139.

Davies, P. T., Harold, T., Goeke-Morey, M. C. \& Cummings, E. M. (2002). Child emotional security and interparental conflict. Monographs of the Society for Research in Child Development, 67(3), vii-viii.

Davies, P. T. \& Lindsay, L. L. (2004). Interparental conflict and adolescent adjustment: Why does gender moderate early adolescent vulnerability? Journal of Family Psychology, 18(1), 160-170.

El-Sheikh, M. \& Reiter, S. L. (1996). Children's responding to live interadult conflict: The role of form of anger expression. Journal of Abnormal Child Psychology, 24(4), 401-415.

Forman, E. M. \& Davies, P. T. (2005). Assessing children's appraisals of security in the family system: The development of the Security in the Family System (SIFS) scales. Journal of Child Psychology and Psychiatry, 46(8), 900-916.

Goeke-Morey, M. C., Cummings, E. M., Harold, G. T. \& Shelton, K. H. (2003). Categories and continua of destructive and constructive marital conflict tactics from the perspective of U.S. and Welsh children. Journal of Family Psychology, 17(3), 327-338.

Grych, J. H. \& Cardoza-Fernandes, I. (2001). Understanding the impact of interparental conflict on children: The role of social cognitive processes. En J. H. Grych \& F. D. Fincham (Eds.), Interparental conflict and child development: Theory, research, and applications (pp. 157-187). New York: Cambridge University Press.

Grych, J. H. \& Fincham, F. D. (1990). Marital conflict and children's adjustment: A cognitive-contextual framework. Psychological Bulletin, 108(2), 267-290.

Grych, J. H., Seid, M. \& Fincham, F. D. (1992). Assessing marital conflict from the child's perspective:
The children's perception of Interparental Conflict Scale. Child Development, 63(3), 558-572.

Jouriles, E. N. \& Norwood, W. D. (1995). Physical aggression toward boys and girls in families characterized by the battering of women. Journal of Family Psychology, 9(1), 69-78.

Justicia, M. J. \& Cantón, J. (2011). Conflicto entre padres y conducta agresiva y delictiva de los hijos. Psicothema, 23(1), 20-25.

Kurdek, L. A. (1986). Children's reasoning about parental divorce. En R. D. Ashmore \& D. M. Brodzinsky (Eds.), Thinking about the family: Views of parents and children (pp. 233-276). New Jersey: LEA.

López Larrosa, S. (2009). El sistema familiar ante el divorcio: factores de riesgo y protección y programas de intervención. Cultura y Educación, 21(4), 391-402.

Luthar, S. S., Doernberger, C. H. \& Zigler, E. (1993). Resilience is not a unidimensional construct: Insights from a prospective study of inner-city adolescents [Special Issue]. Development and Psychopathology, 5(4), 703-717.

Lytton, J. (1991). Parents' differential socialization of boys and girls: A meta-analysis. Psychological Bulletin, 109(2), 267-296.

Martínez-Pampliega, A. (2008). Escala de Conflicto Interparental desde la Perspectiva de los/as Hijos/ as (CPICS. The Children's perception of Interparental Conflict Scale). En Equipo EIF, Manual de instrumentos de evaluación familiar (pp. 143-152). Madrid: CCS.

Rodrigo, M. J., Máiquez, M. L., Padrón, I. \& García Ruiz, M. (2009). ¿Por qué y con qué intención lo hizo? Atribuciones de los padres y adolescentes en los conflictos familiares. Psicothema, 21(2), 268-273.

Rutter, M. (1990). Commentary: Some focus and process considerations regarding the effects of parental depression on children. Developmental Psychology, 26(1), 60-67.

Sánchez Souto, V., López Larrosa, S. \& Mendiri, P. (2010, julio). Propiedades psicométricas de la escala de Conflicto Interparental desde la Perspectiva de los Hijos (CPIC) y de la escala de Seguridad en el Sistema Familiar (SIFS). Documento presentado en VII Congreso Iberoamericano de Psicología, Oviedo, España. 